\title{
CASE STUDY AND LITERATURE REVIEW
}

\section{Posttraumatic Tophaceous Gout: A Case Report and Literature Review}

\author{
Richard Simman, MD, CWS, FACS, FACCWS ${ }^{a, b, *}$, Brent Kirkland, MDc,d, \\ Sarah Jackson, DOd
}

\author{
${ }^{a}$ Boonshoft School of Medicine, Wright State University, Miamisburg, OH 45342, USA \\ ${ }^{b}$ Sycamore Wound Center at Sycamore Medical Center, Miamisburg, OH 45342, USA \\ ${ }^{c}$ Stanford University Department of Dermatology Residency Program, Palo Alto, CA, 94305, USA \\ ${ }^{d}$ Kettering Medical Center Internal Medicine Residency Program, Kettering, OH 45429, USA
}

\section{KEYWORDS: \\ Gout; \\ Tophus; \\ Traumatic; \\ Biopsy; \\ Nonhealing wound}

\begin{abstract}
In the field of wound care, the management of nonhealing wounds requires particular attention. Biopsy is often performed in such cases to rule out underlying malignancy; however, the differential remains broad. In the following case, we discuss a nonhealing post traumatic wound in a diabetic patient, secondary to unrecognized tophus.

(C) 2009 Elsevier Inc. All rights reserved.
\end{abstract}

\section{Introduction}

In the field of wound care, the management of nonhealing wounds requires particular attention. Biopsy is often performed in such cases to rule out underlying malignancy; however, the differential remains broad. In the following case, we discuss a nonhealing post traumatic wound in a diabetic patient, secondary to unrecognized tophus.

\section{Case Report}

An 84-year-old woman presented to our wound center for the evaluation of nonhealing wound on the tip of the

Conflict of interest: The authors report no conflicts of interest.

* Corresponding author.

E-mail address: plasticsimman@yahoo.com second toe of her left foot. The patient recalled injuring the toe approximately 6 months prior, having scraped the toe against the ground at that time. Otherwise, she noted no recurrent injury to the area and no resolution despite daily dressing changes and the use of topical triple antibiotic ointment. The patient denied any preexisting lesion at the involved toe prior to the injury. Her medical history

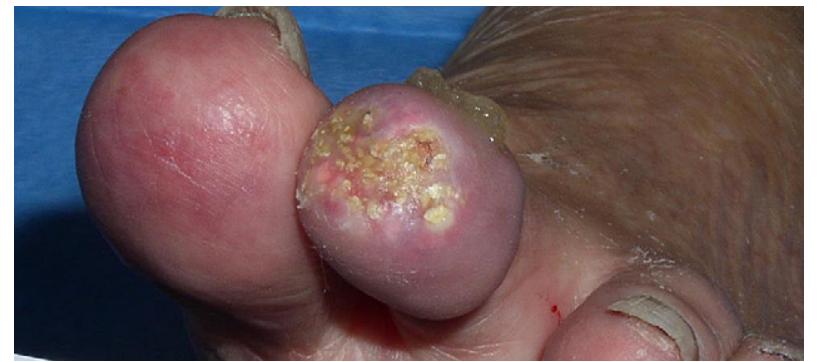

Figure 1 Preprocedure Photo of Left Second Toe at First Presentation. 


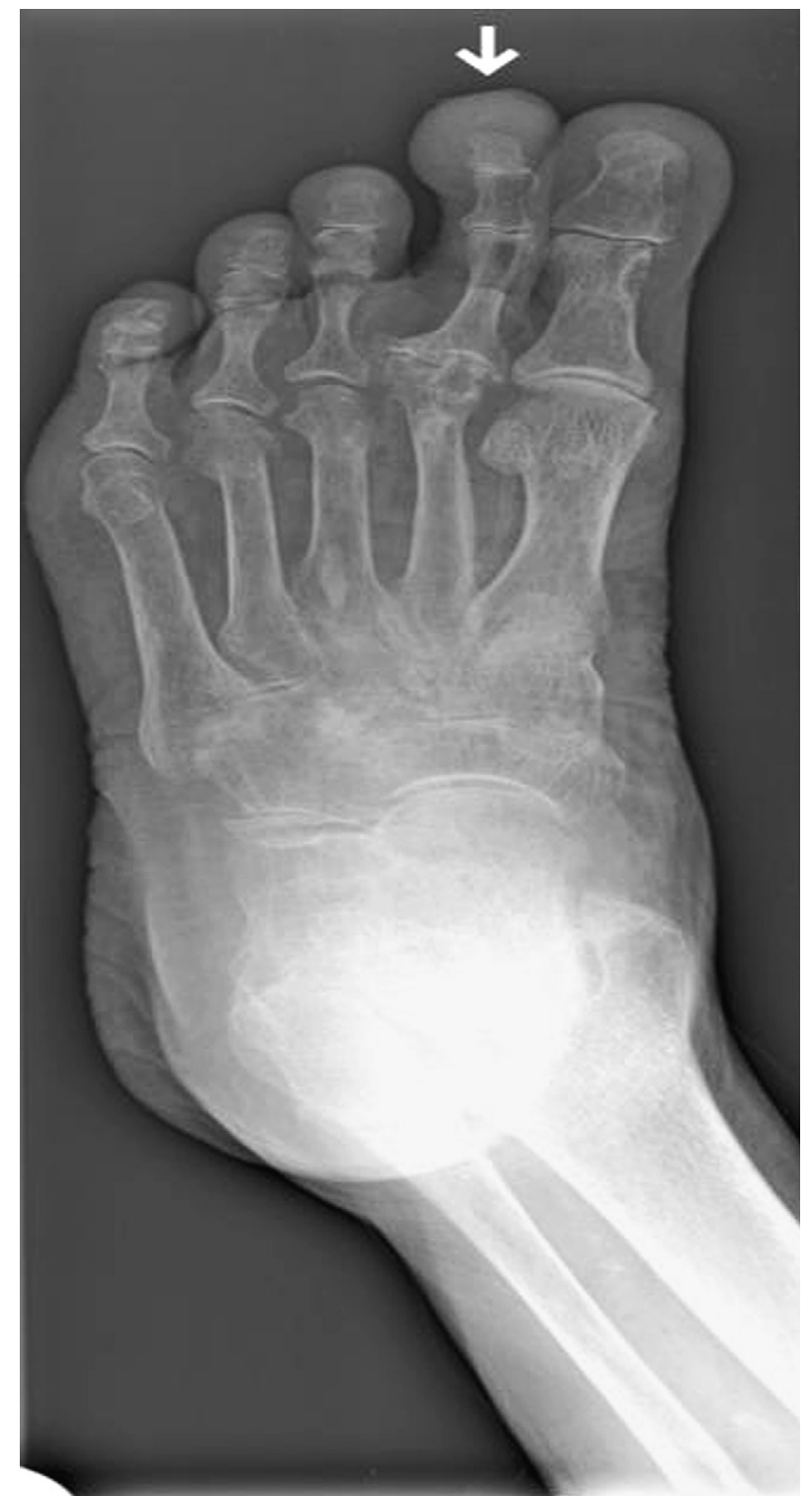

Figure 2 X-ray of Left Foot Showing Old Fracture at the Head of the Second Metatarsal; Arrow Indicates Soft Tissue Swelling at the Tip of the Second Toe. The X-ray also showed an old fracture at the base of the second toe.

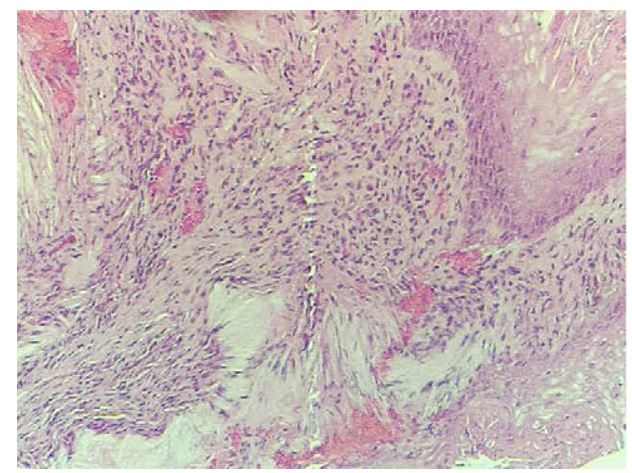

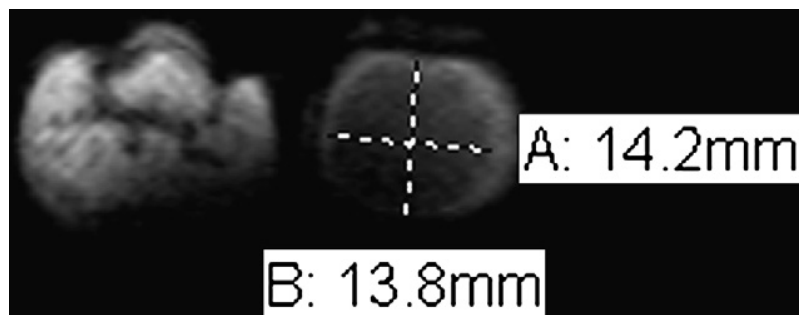

Figure 3 MRI Image of the Lesion Measuring $14.2 \times 13.8 \mathrm{~mm}$.

included type I diabetes mellitus, congestive heart failure, and hypertension, all of which were being adequately managed according to the patient's history.

On physical examination, the second toe of the left foot demonstrated an erythematous, crusted lesion on the distal aspect of the toe not involving the nail (Figure 1).

The patient's distal pulses including dorsalis pedis and posterior tibialis were palpable on examination.

X-ray of the left foot showed soft tissue swelling of the tuft of the left second toe (Figure 2). Radiology recommended magnetic resonance imaging (MRI) for further evaluation of possible osteomyelitis. MRI showed a $1.4 \times 1.4-\mathrm{cm}$ well-defined soft tissue mass of the tuft of the second toe, demonstrating low signal intensity on $\mathrm{T} 1$ and intermediate signal intensity on $\mathrm{T} 2 /$ short $\mathrm{T} 1$ inversion recovery (Figure 3).

The image did not enhance with gadolinium administration or invade the adjacent structures. Initial laboratory results showed normal renal function and a uric acid level of 7.3 mg per dL (normal value: 2.6-7.2 mg per $\mathrm{dL}$ ). A biopsy was also performed at the patient's initial visit to rule out underlying carcinoma. The resulting pathology revealed uric acid crystal formation consistent with tophaceous gout (Figure 4).

Treatment included digital block followed by evacuation of the soft tissue mass, which was noted to have a very friable consistency. The excision site was closed with sutures and dressed with sterile ointment and gauze. The patient returned 1 week later for postprocedural follow-up,

Figure 4 Pathology of Punch Biopsy of Second Digit of Left Toe Showing Amorphous Crystalline Deposits and Inflammation Consistent With Gouty Tophus $(\times 100)$. 


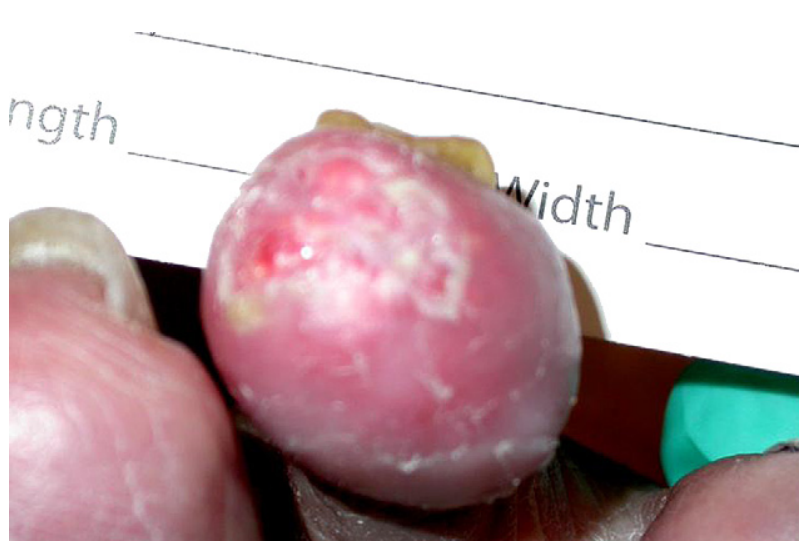

Figure 5 View of Second Toe 2 Months Postprocedure, Showing Complete Epithelialization and Less Swelling.

and the site of the lesion appeared to be well healed (Figure 5). The patient was started on allopurinol for prevention of secondary recurrence and was referred to rheumatology to rule out any additional systemic involvement of disease.

\section{Discussion}

Gout is a common metabolic problem that results from improper purine metabolism. Although the initial presentation typically includes arthritis, gouty tophi can also appear at the onset of disease. Uric acid crystals deposit in the skin and soft tissues, leading to the formation of tophi. These tophi typically localize to the extremities. Despite the idea that tophi represent a more chronic course of gout, they can also present at the initial time of diagnosis despite a lack of joint findings. ${ }^{1}$

Tophi also tend to appear more frequently in older people than in younger populations. Risk factors for older patients include the use of low-dose aspirin, alcohol abuse, decreased renal function, and prolonged use of diuretics. ${ }^{2}$

The serum levels of uric acid do not always correlate with the clinical picture. Acute gouty attacks may still occur with normal serum urate levels. ${ }^{3}$ In this patient, a diagnosis of tophaceous gout was particularly challenging based on history and physical examination alone because the patient had no prior history of gout and the lesion assumed an atypical dermatologic manifestation. Most commonly, tophi present as firm pink nodules or swelling. ${ }^{4}$ This patient lacked these findings on physical examination. Other presentations may include yellow or erythematous lesions, ulcerations, or even hyperkeratotic skin findings mimicking squamous cell carcinoma. ${ }^{5}$ Because the wound failed to heal, biopsy was indicated to exclude underlying carcinoma.

From review of the literature, this is the first reported case of a nonhealing wound secondary to post-traumatic development of tophaceous gout. Surgical management can result in delayed wound healing with regard to tophaceous gout at the distal interphalangeal joint; however, this case did not present with involvement of the joint. ${ }^{6}$ Furthermore, trauma rather than surgery was the inciting event. We believe that the regional inflammation caused by the injury may have triggered the precipitation of soft tissue tophaceous gout. One case report attempting to identify the clinical course of intradermal tophi described 5 patients with intermittent tophus ulcerations. Findings did not include delayed wound healing. Additionally, drainage of chalky white material was described in these cases. ${ }^{7}$ This particular clinical finding did not manifest in this patient. Considering the numerous possible causes of delayed wound healing, this case reiterates the need for biopsy in patients with chronic, nonhealing wounds.

\section{References}

1. Thissen CA, Frank J, Lucker GP: Tophi as first clinical sign of gout. Int J Dermatol. 2008;47(suppl 1):49-51.

2. Fam AG: Gout, diet, and the insulin resistance syndrome. J Rheumatol. 2002;29(7):1350-1355.

3. Schlesinger N, Norquist JM, Watson DJ: Serum urate during acute gout. J Rheumatol. 2009;36(6):1287-1289.

4. Touart DM, Sau P. Cutaneous deposition diseases. Part II. J Am Acad Dermatol. 1998;39(4 Pt 1):527-544. [quiz: 545-546].

5. Dacko A, Hardick K, McCormack P, Szaniawski W, Davis I: Gouty tophi: a squamous cell carcinoma mimicker? Dermatol Surg. 2002;28(7): 636-638.

6. Mudgal CS: Management of tophaceous gout of the distal interphalangeal joint. J Hand Surg [Br]. 2006;31(1):101-103.

7. Fam AG, Assaad D: Intradermal urate tophi. J Rheumatol. 1997;24(6): $1126-1131$. 